\title{
EFFECTIVENESS OF TRAINING INTERVENTIONS ON THE HAZARD ANTICIPATION FOR YOUNG DRIVERS DIFFERING IN SENSATION SEEKING BEHAVIOR
}

\author{
Tingru Zhang ${ }^{1}$, Krishna Valluru ${ }^{1}$, Tracy M. Zafian ${ }^{1}$, Yusuke Yamani ${ }^{2}$, \& Siby Samuel ${ }^{1}$ \\ ${ }^{1}$ University of Massachusetts Amherst \\ ${ }^{2}$ Old Dominion University \\ Email: tingruzhang@acad.umass.edu
}

\begin{abstract}
Summary: Drivers younger than 25 years are overrepresented in fatal crashes compared to experienced drivers between 30 and 55 years of age. This age-related difference in crash statistics partly arises from younger drivers' poor hazard anticipation. Training programs (e.g. SAFE-T; Yamani et al. (2016)) have been shown effective at improving these drivers' anticipation behavior. However, individual differences such as sensation-seeking behavior, aggression, and driving violations exist in young drivers and may contribute to differences in their hazard anticipation. The current driving simulator study examined whether three individual differences known to characterize driving behavior can predict hazard anticipation performance for young drivers, and training effectiveness. K-mean clustering technique classified participants into two clusters based on their driving aggression, sensation seeking and driving violation scores. The results indicated that the low sensation-seeking drivers benefitted more from the training than their high sensation-seeking peers. These findings have design implications for the development of appropriate countermeasures for high sensation-seeking drivers.
\end{abstract}

\section{INTRODUCTION}

Young drivers below the age of 25 are at a higher crash risk compared with older, experienced drivers aging between 30 and 55 years. Most non-fatal accidents involving young drivers result from cluelessness rather than carelessness (McKnight \& McKnight, 2003). Cluelessness represents the failure to recognize the dangers while driving due to the lack of awareness while carelessness indicates a propensity to intentionally speed or deliberately drive recklessly. Data indicate that the top crash contributing behaviors for young driver crashes are errors in attention and visual search, rather than deliberate risk-taking behaviors (McKnight \& McKnight, 2003).

Young drivers are poor at anticipating hazards (Horswill \& McKenna, 2004; Underwood et al., 2002). Training programs have been developed that have been proven effective at improving young drivers' hazard anticipation skills both on a driving simulator and on road (Crundall et al., 2010; Pradhan et al., 2009). Drivers trained on such programs have been found to retain these skills for up to a year following training in a field evaluation (Taylor et al., 2011). However, the literature clearly notes that young drivers are not a homogenous group and differ in their drivingrelated attitudes, personality traits, hazard perception ability and aggression (Deery \& Fildes, 1999), potentially compromising the effectiveness of such training programs. That is, all young drivers are not the same and therefore, may not uniformly benefit from such training. 
There is evidence that deliberate risk taking behaviors are associated with high sensation-seeking personality (Beirness, 1996) and high driving aggression (Dahlen et al., 2005; Lajunen \& Parker, 2001). Given the above, training that is shown to be effective for non-aggressive drivers may not be as effective for aggressive, risk-seeking drivers. The purpose of this driving simulator study is to examine whether young drivers differing in sensation-seeking behavior, aggression, and violation scores benefit equally from an existing training program designed to improve novice drivers' hazard anticipation performance. We hypothesize that drivers with a low risk-seeking tendency will in general anticipate a greater proportion of latent hazards after training than the drivers with high risk-taking propensity.

\section{METHOD}

\section{Experimental design and procedures}

This study employed a between-subject design, with one group of participants doing a computerbased driver training program called SAFE-T, which includes performance feedback, and the rest of the participants, the control group, being given a placebo training program.

Each participant completed one session lasting approximately 75 minutes. At the beginning of each session, they completed questionnaires on their demographic characteristics, driving history and driving behavior. Participants were assigned to receive either the SAFE-T training or placebo training (30-40 minutes). After the training participants were fitted with the eye tracker and then drove on the simulator through four drives (4-6 minutes each) with two scenarios each. They were required to drive and behave as they normally do in real-life.

\section{Questionnaires}

The first questionnaire collected demographic information such as age, gender and driving experience. Three additional questionnaires measured participants' risk-seeking behavior. The Arnett Inventory of Sensation Seeking (AISS) questionnaire (Arnett, 1994) measures sensation seeking. Participants indicated how much each of 20 items applied to them on a 4-point Likert scale (1= "Describes me very well"; $4=$ "Does not describe me at all"). The Aggressive Driving Tendency (ADT) questionnaire (Snow, 2000) measures driving aggression tendencies. Participants rated their frequency of the 17 listed aggressive behaviors on a 4-point scale $(1=$ "Never" to 4= "Often"). The ordinary violation subscale, in total 16 items, from the Driver Behavior Questionnaire (DBQ) measures aberrant driving behaviors. There is evidence that DBQ scores are related to sensation seeking (Schwebel et al., 2006) and driving aggression personalities and are a predictor of traffic crashes (De Winter \& Dodou, 2010).

\section{Training}

$S A F E-T$. The SAFE-T program consists of 4 training components: one module each designed to improve young drivers' skills in hazard anticipation, hazard mitigation, and attention maintenance, and a review section to go over the main skills taught in each training module. The SAFE-T training, presented in Microsoft PowerPoint, uses an error-based learning approach: trainees who make any mistakes during a training module are shown what they could have done differently for better safety and then given the chance to practice those skills. For additional details, SAFE-T is described more thoroughly in Hamid et al. (2016). 


\section{Placebo training}

The placebo training is designed to look like other driver training programs, but provides no feedback and no instruction on how to anticipate and respond to latent hazards.

\section{Participants}

A total of 49 drivers ranging from 18 to 24 years of age participated in the study. The drivers were pseudo-randomly assigned to either the control placebo training group $(N=23 ; 12$ females $)$ or the SAFE-T training group $(N=26 ; 14$ females). The two groups of participants were of similar age (Placebo group: mean age $=21.2$ years, $S D=1.73$; SAFE-T group: mean age $=20.5$ years, $S D=1.53 ; \mathrm{F}(1,45)=2.13, p=0.22$, and with similar levels of driving experience (Placebo group: mean $=42.5$ months since licensure, $S D=28.75$; SAFE-T group: mean $=36.8$ months, $S D=22.49 ; \mathrm{F}(1,45)=0.59, p=0.44)$.

\section{Apparatus}

Simulator. A STSTIM three-channel driving simulator system from Systems Technology Inc. was used for this study. The system includes a driver's seat, a steering wheel and pedal console, and three $60^{\prime \prime}$ screens in front of the driver, subtending at least $160^{\circ}$ of visual angle.

Eye Tracker. Participants' eye movements during their simulator drives were recorded using a MobileEye XG head-mounted eye tracker from Applied Science Laboratories (ASL), with a visual range of $50^{\circ}$ horizontal and $40^{\circ}$ vertical, and accuracy of $0.5^{\circ}$ of visual angle.

\section{Simulator drive scenarios and scoring}

Each drive contained two scenarios for evaluating participants' hazard anticipation skills. The eye tracking videos for each of these scenarios were scored by two independent scorers who were blind to the treatment conditions. Participants' eye movements for a scenario were binary scored as a 1 (hit) if a participant took a glance towards the pre-determined target zone while in the launch zone (and 0 otherwise). As defines in previous studies, the target zone here is defined as the area of the roadway that the driver should scan to anticipate a potential hazard. The launch zone is defined as that area of the roadway where the driver should begin glancing at the target zone to be able to successfully anticipate and mitigate the threat (Yamani et al., 2016).

\section{Statistical analysis}

Young drivers were first classified into different groups based on their sensation-seeking, driving aggression, and driving behavior scores. The advantages of cluster analysis, compared with median-splits, are that it takes the three dimensions into consideration to give classifying results and the number of clusters is not limited to two. The best number of clusters was determined using the partitioning around medoids (PAM) clustering technique and the clustering results was generated by the K-means cluster algorithm. ANOVA and linear regression was used to investigate the effect of training and personalities on drivers' hazard anticipation skills after training (SAFE-T or placebo).

\section{RESULTS}

When examining the questionnaires, the average score was $2.59(S D=0.42), 1.44(S D=0.37)$, and $1.43(S D=0.51)$ for the sensation seeking, driving aggression and aberrant driving scales, 
respectively. No significant score differences were found between the placebo and SAFE-T groups.

\section{Classification}

The partitioning around medoids clustering suggested the most appropriate number of clusters was two. The K-mean clustering results (Figure1) showed that $80.7 \%$ of the point variance was explained by the two clusters. As shown in Figure 1a, 20 participants (14 from placebo group and 16 from SAFE-T group) were classified into Cluster 1 . The remaining 19 participants (10 from placebo and 9 from SAFE-T group) were classified into Cluster 2. The aggressive driving score was at lower levels for both clusters. However, compared with Cluster 1 (Figure 1b), Cluster 2 is characterized by a higher score on the Sensation Seeking, and Driving Violations scale. Given that the difference is largest on the sensation seeking scale, the two clusters were named as "low sensation-seeking" and "high sensation-seeking" drivers, respectively.

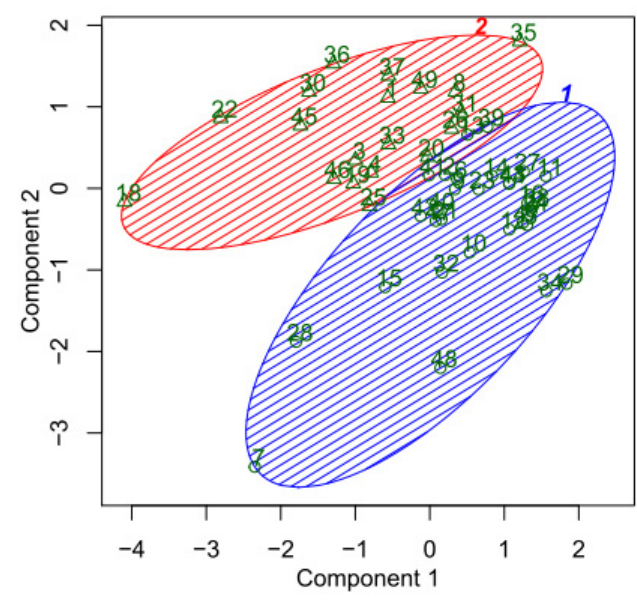

a. 2D Representation of the Cluster Solution

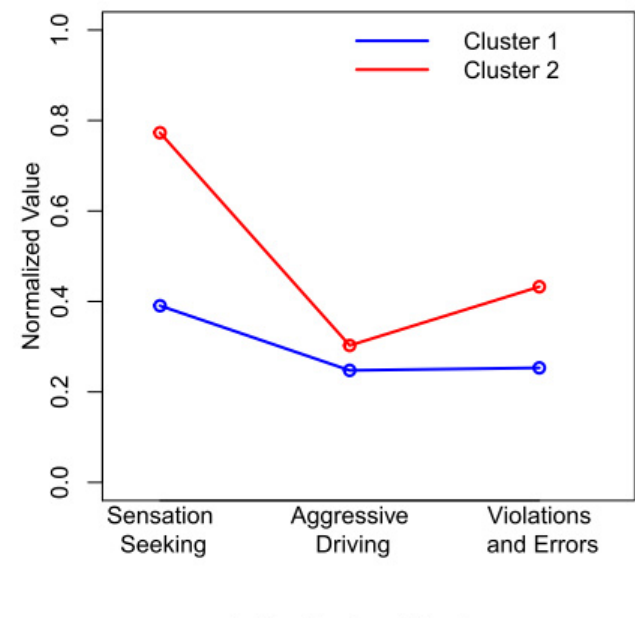

b. The Center of Clusters

Figure 1. The K-mean classification results. Left: The scatter plot of the first two principal components that were derived from the data. Right: The center of each cluster.

\section{Hazard anticipation performance}

Eye tracking data from two participants, both belonging to the SAFE-T group, were lost due to equipment malfunction. The data for the remaining forty-seven participants were analyzed for hazard anticipation.

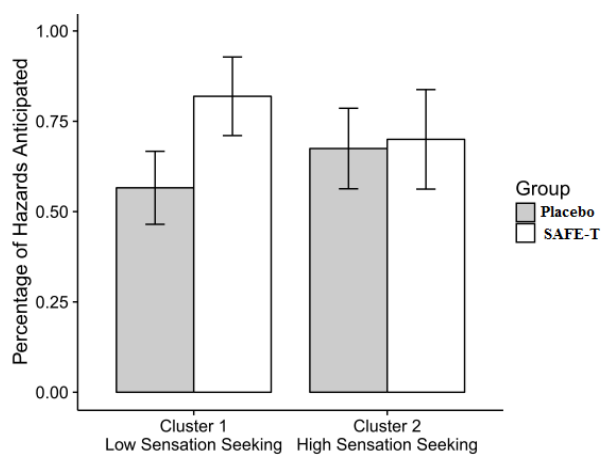

Figure 2. The percentage of hazards anticipated by each cluster. (Each error bar represents the between-participant $95 \%$ confidence interval.) 
First, proportions of the events with correct hazard anticipation were submitted to a $2 \times 2$ between-participant analysis of variance (ANOVA) with Training (SAFE-T vs. Placebo) and Cluster (High vs. Low sensation-seeking) as between-participant factors. Figure 2 presents proportions of hazards correctly anticipated. The results indicate that proportions of hazards anticipated were greater among the SAFE-T trained drivers than the Placebo-trained drivers, replicating the effectiveness of the SAFE-T program $\left[F(1,43)=10.69, \eta^{2}=0.20, p=.002\right)$. The main effect of Cluster was not statistically significant $[p=.94]$. However, their interaction effect was statistically significant $\left[F(1,43)=4.37, \eta^{2}=0.09, p=.043\right]$. Follow-up t-tests with the Bonferroni correction for the critical alpha value further revealed that the SAFE-T improved drivers' hazard anticipation performance for the low sensation-seeking drivers [independentsamples $t(28)=3.66, p=.001$ ], but did not for the high sensation-seeking drivers [independentsamples $t(15)=0.34, p=.742]$.

\section{DISCUSSION}

Compared to middle-aged and experienced drivers, teenage (16 to 17 years old) and young (19 to 29 years old) drivers have been found to be more likely to fail to anticipate potential hazards on the roadway (Pradhan et al., 2005). Our results are consistent with this; the placebo group participants successfully only anticipated about 50 percent of the latent hazards. Recent studies attribute the 'cluelessness' of novice drivers as the main reason for this poor performance (McKnight \& McKnight, 2003), that is, teen drivers are unaware of where to scan for hazards due to their limited exposure to risky scenarios. However, we cannot entirely rule out the possibility that a small proportion of these are careless, that is, they purposely ignore such hazards even when aware of them. This study demonstrated that only drivers with a low desire for seeking risks showed significant performance improvements following training. Even though the high sensation-seeking trained drivers were explicitly trained on scanning towards the location of the hazards, and made aware of the potential risks of failing to anticipate hazards, they were reluctant to actively search for the hazards during the evaluation drives. One reason to explain why high sensation seekers are willing to take more risks may be due to the optimistic bias (Weinstein, 1980) present in high sensation seekers that makes them perceive themselves to be less at risk. The finding that a high propensity of seeking sensation in young drivers can undermine the effectiveness of training suggests that training itself is not sufficient to improve their hazard anticipation performance of certain young drivers. Compounding this issue is that fact, while driving skill may improve with driving experience, the desire for sensation-seeking endures and even increases among male drivers from age 21 to age 26 (Begg \& Langley, 2001).

While the glancing behaviors significantly improved with training, it remains unclear how the actual driving behavioral adaptation will be modified with training. In a follow-up analysis, we will investigate whether successful anticipation transfers to appropriate mitigating behaviors (e.g. slowing down when approaching the hazards). Another important question that is not investigated here is the impact of improved skill on drivers' future risk-taking attitude. It is possible that an increase in risk taking could follow skill-based training as a result of an increase in self-confidence (Krueger \& Dickson, 1994). However, McKenna et al. (2006) argued that a decrease in risk taking is associated with the hazard anticipation training. It should be clear that this is a general conclusion without taking personality factors into account. Given what we found 
here, it is necessary for future studies to re-evaluate how training may affect drivers' risk-taking attitude and how the effect could be moderated by the sensation-seeking personality.

In this study, the evaluation was administered immediately after the training, which may raise concern about the practical question of whether the training is likely to have a longer-term effect and whether the improved hazard anticipation skill can be transferred to actual crash reduction. The answers to both concerns are yes, as it has been found that the hazard anticipation difference between untrained and trained drivers was still significant six months or longer after training (Taylor et al., 2011) and there was a significant reduction in crashes within the one year period following training (Zhang et al., 2016). Based on our findings, there exists a premise for traffic safety researchers as well as the government to consider appropriate policy-based countermeasures or augmented training interventions that may reduce the crash risk of those young drivers who are more risk-taking. This group of drivers is at a higher crash risk yet training has been proven to have little effect on them.

There are some limitations of this study. One is that the evaluation was carried out on a simulator and drivers may behave differently compared with in a field test or in naturalistic condition. Another limitation is that no gender effect was investigated although there is evidence in literature that the training is only effective for male young drivers (Thomas et al., 2016).

\section{REFERENCES}

Arnett, J. (1994). Sensation seeking: A new conceptualization and a new scale. Personality and individual differences, 16(2), 289-296.

Begg, D., \& Langley, J. (2001). Changes in risky driving behavior from age 21 to 26 years. Journal of safety research, 32(4), 491-499.

Beirness, D. (1996). The relationship between lifestyle factors and collisions involving young drivers. Paper presented at the New to the road: Reducing the risks for young motorists. Proceedings of the First Annual International Symposium of the Youth Enhancement Service.

Crundall, D., Andrews, B., Van Loon, E., \& Chapman, P. (2010). Commentary training improves responsiveness to hazards in a driving simulator. Accident Analysis \& Prevention, 42(6), 2117-2124.

Dahlen, E. R., Martin, R. C., Ragan, K., \& Kuhlman, M. M. (2005). Driving anger, sensation seeking, impulsiveness, and boredom proneness in the prediction of unsafe driving. Accident Analysis \& Prevention, 37(2), 341-348.

De Winter, J., \& Dodou, D. (2010). The Driver Behaviour Questionnaire as a predictor of accidents: A meta-analysis. Journal of safety research, 41(6), 463-470.

Deery, H. A., \& Fildes, B. N. (1999). Young novice driver subtypes: Relationship to high-risk behavior, traffic accident record, and simulator driving performance. Human Factors: The Journal of the Human Factors and Ergonomics Society, 41(4), 628-643.

Hamid, M., Samuel, S., Borowsky, A., Horrey, W. J., \& Fisher, D. L. (2016). Evaluation of Training Interventions to Mitigate Effects of Fatigue and Sleepiness on Driving Performance. Paper presented at the Transportation Research Board 95th Annual Meeting.

Horswill, M. S., \& McKenna, F. P. (2004). Drivers' hazard perception ability: Situation awareness on the road. 
Krueger, N., \& Dickson, P. R. (1994). How believing in ourselves increases risk taking: Perceived self-efficacy and opportunity recognition. Decision Sciences, 25(3), 385-400. Lajunen, T., \& Parker, D. (2001). Are aggressive people aggressive drivers? A study of the relationship between self-reported general aggressiveness, driver anger and aggressive driving. Accident Analysis \& Prevention, 33(2), 243-255.

McKenna, F. P., Horswill, M. S., \& Alexander, J. L. (2006). Does anticipation training affect drivers' risk taking? Journal of Experimental Psychology: Applied, 12(1), 1.

McKnight, A. J., \& McKnight, A. S. (2003). Young novice drivers: careless or clueless? Accident Analysis \& Prevention, 35(6), 921-925.

Pradhan, A. K., Hammel, K. R., DeRamus, R., Pollatsek, A., Noyce, D. A., \& Fisher, D. L. (2005). Using eye movements to evaluate effects of driver age on risk perception in a driving simulator. Human Factors: The Journal of the Human Factors and Ergonomics Society, 47(4), 840-852.

Pradhan, A. K., Pollatsek, A., Knodler, M., \& Fisher, D. L. (2009). Can younger drivers be trained to scan for information that will reduce their risk in roadway traffic scenarios that are hard to identify as hazardous? Ergonomics, 52(6), 657-673.

Schwebel, D. C., Severson, J., Ball, K. K., \& Rizzo, M. (2006). Individual difference factors in risky driving: The roles of anger/hostility, conscientiousness, and sensation-seeking. Accident Analysis \& Prevention, 38(4), 801-810.

Snow, R. W. (2000). 1999 national highway safety survey: monitoring Americans' attitudes, opinions, and behaviors.

Taylor, T. G., Masserang, K. M., Pradhan, A. K., Divekar, G., Samuel, S., Muttart, J. W., . . . Fisher, D. L. (2011). Long term effects of hazard anticipation training on novice drivers measured on the open road. Paper presented at the Proceedings of the... International Driving Symposium on Human Factors in Driver Assessment, Training, and Vehicle Design.

Thomas, F. D., Rilea, S., Blomberg, R. D., Peck, R. C., \& Korbelak, K. T. (2016). Evaluation of the safety benefits of the risk awareness and perception training program for novice teen drivers: Washington, DC: National HIghway Traffic Safety Administration.

Underwood, G., Chapman, P., Bowden, K., \& Crundall, D. (2002). Visual search while driving: skill and awareness during inspection of the scene. Transportation Research Part F: Traffic Psychology and Behaviour, 5(2), 87-97.

Weinstein, N. D. (1980). Unrealistic optimism about future life events. Journal of personality and social psychology, 39(5), 806.

Yamani, Y., Samuel, S., Knodler, M. A., \& Fisher, D. L. (2016). Evaluation of the effectiveness of a multi-skill program for training younger drivers on higher cognitive skills. Applied Ergonomics, 52, 135-141.

Zhang, T., Li, J., Thai, H., Zafian, T., Samuel, S., \& Fisher, D. L. (2016). Evaluation of the Effect of a Novice Driver Training Program on Citations and Crashes. Paper presented at the Proceedings of the Human Factors and Ergonomics Society Annual Meeting, September 1923, 2016, Washington DC. 